\title{
DISEÑO DE UNA NUEVA PINZA ROBÓTICA DE RESISTENCIA VARIABLE USANDO DEDOS DE PARTÍCULAS
}

\author{
Daniel Cardin-Catalan, Angel P. del Pobil and Antonio Morales \\ Robotic Intelligence Laboratory, Universitat Jaume I \\ (cardin, pobil, morales)@uji.es
}

\begin{abstract}
Resumen
Este artículo aborda el diseño de una pinza de rigidez variable. Esta se utiliza para resolver un problema que surge en las tiendas en línea, en las que grandes almacenes mantienen todo el stock. En el proceso de automatización de la tarea de escoger objetos individuales, un agarre confiable es un problema importante debido a la incertidumbre de la forma y posición de los objetos. Para abordar este problema, se ha diseñado una nueva pinza de rigidez variable capaz de agarrar diferentes tipos de objetos mediante el uso y la adaptación de la tecnología del Universal Gripper en una pinza paralela de 2 dedos. Para probar la pinza, se han manipulado cinco objetos diferentes, y se compararon con otro diseño de pinza simple basado en el Festo ${ }^{\circledR}$ FinGripper. Los experimentos muestran que esta pinza, con accionamiento de rigidez variable, mejora las capacidades de agarre de una pinza sin la capacidad de rigidez variable.
\end{abstract}

Palabras clave: Resistencia variable, Pinza Robótica, Partículas.

\section{INTRODUCCIÓN}

Durante los últimos años, han aparecido grandes tiendas minoristas en línea (Amazon, GearBest, Ocado, etc.) que han logrado un gran aumento y expansión en todo el mundo. Como resultado de este crecimiento, estas compañías necesitan enormes almacenes para mantener todo el stock necesario para las compras en línea. Los sistemas de robótica y automatización están comenzando a utilizarse para mejorar la productividad dentro de los almacenes, pero solo recientemente este enfoque de automatización consideró el paso en el que los productos se toman de los estantes y se colocan en la caja que se enviará al comprador. Este último paso debe ser lo más rápido posible, debido a la alta demanda de objetos de los almacenes.

Amazon, uno de los almacenes de venta online más grandes, organiza desde 2015 el Amazon Robotic Challenge, donde invitan a grupos de investigación

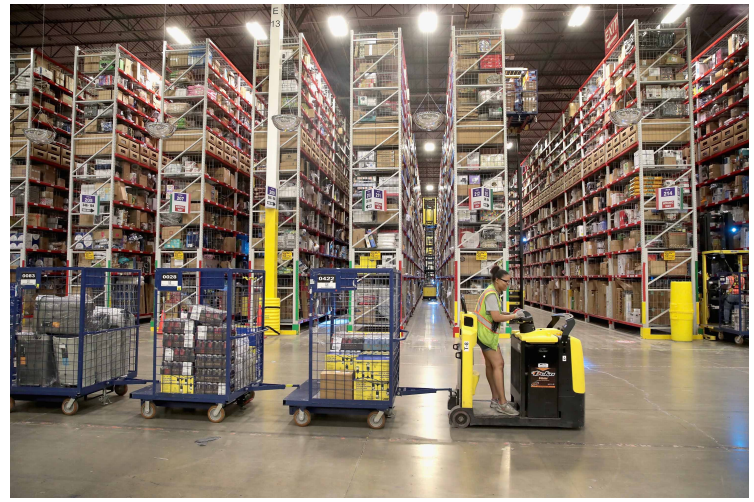

Figura 1: Almacén de Amazon

en robótica de todo el mundo. El objetivo principal de esta competencia es tratar de resolver el problema de automatizar el proceso de colocar objetos en las cajas de venta al cliente. De nuestra participación en las ediciones 2015 y 2017 [5] aprendimos que se necesita una pinza adaptable cuando solo está disponible la apariencia de los objetos sin ninguna o poca información previa de ellos. Por lo que la pinza debe superar el software y controlar posibles errores.

Hoy en día, en las industrias pesadas, las pinzas robóticas tradicionales o convencionales son las más utilizadas, debido a su precisión y velocidad de agarre. Sin embargo, necesitan algunos elementos externos, como el control de la visión o la retroalimentación de fuerza para poder desarrollar un agarre adaptable [4]. Esta adición de elementos externos o elementos de accionamiento adicionales, como utilizar un motor por cada grado de libertad, para conseguir que las pinzas rígidas sean adaptables generalmente hace que sean bastante pesadas o caras.

La robótica blanda o Soft Robotics, pareció resolver el problema principal de adaptación de las pinzas rígidas o tradicionales, esta robótica abarca la sección que trata con robots que están hechos con materiales blandos o los que interactúan con objetos blandos o desconocidos a priori $[7,9]$.

Para resolver el problema principal descrito anteriormente, es decir, agarrar objetos desconoci- 
dos de manera confiable, se propone el diseño y la fabricación de una pinza de rigidez variable. La pinza está compuesta por dos dedos que forman un agarre paralelo con un grado de libertad; este enfoque de agarre se selecciona porque es el tipo de agarre más simple, por lo que simplifica el control para agarrar y manipular los objetos. La rigidez se puede cambiar gracias a su propia estructura de dedos. La estructura es similar a la utilizada por el Universal Gripper [10], es decir, utilizando una presión negativa para crear un estado duro para los dedos blandos. En la fig. 2, la pinza propuesta se muestra montada en un brazo robótico.

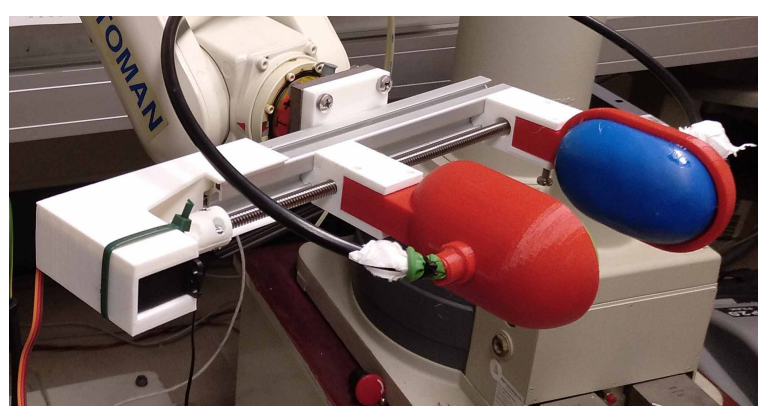

Figura 2: Pinza propuesta

\section{ESTADO DEL ARTE}

Investigaciones recientes $[1,2,8,11,17]$ trabajan sobre pinzas que pueden cambiar su propia rigidez a voluntad, para que puedan lograr más capacidades de agarre o pueden desplegar diferentes fuerzas de agarre según el objeto que vayan a manipular. Algunos de estos enfoques utilizan materiales que pueden cambiar su propia rigidez dependiendo de la temperatura ambiente, normalmente modelados como articulaciones de dedo. Pero necesitan algo de tiempo para que la fuente de calor externa obtenga la temperatura deseada en el material, con lo que este método no es adecuado para el uso en un almacén automatizado, donde el tiempo es crucial. Por otro lado, algunos enfoques utilizan elementos externos como resortes o imanes para crear fuerzas en la actuación principal. Ambos enfoques tienen puntos débiles que un mejor diseño de rigidez variable debería superar, por lo que nuestra pinza debería cambiar la rigidez casi al instante, mientras mantiene un diseño simple, sin la adición de elementos adicionales para cambiar su propia rigidez.

La pinza que llamó especialmente la atención en esta investigación fue el Universal Gripper. El Universal Gripper [10] funciona gracias a la interferencia del material granular dentro de una cubierta de plástico sellada. Cuando se aplica presión negativa en la cavidad plástica con el material granular en el interior gracias a la fricción entre las partículas internas del material y la forma de la cavidad la pinza pasa de un estado blando a uno rígido En consecuencia, si primero se adapta la cubierta a la forma del objeto, cuando se aplica presión negativa, mantendrá la forma del objeto y realizará un agarre estable.

Desde la creación de las pinzas universales, las investigaciones han ido mejorado el diseño y aplicaciones de la misma. Por ejemplo, cambiar el fluido interno para aumentar su rendimiento [13], hacer que funcione con presión positiva en lugar de vacío [10], usarlo para el cálculo de agarre [15] e incluso hacer matrices de pequeños palos fabricados como la pinza universal para realizar agarres [3]; con estas mejoras, se puede utilizar en diversos entornos como las operaciones bajo el agua [12].

En la fig.3 se muestran algunas pinzas de rigidez variable. La primera que se muestra (arriba a la izquierda) es la pinza universal [10]. La segunda (arriba a la derecha) es una pinza que cambia su rigidez con la ayuda de un elemento externo, imanes permanentes [2]. Las de abajo son los que cambian la rigidez utilizando materiales dependientes de la temperatura, el izquierdo se usa con un dedo blando [17] y el derecho con un dedo duro [1].
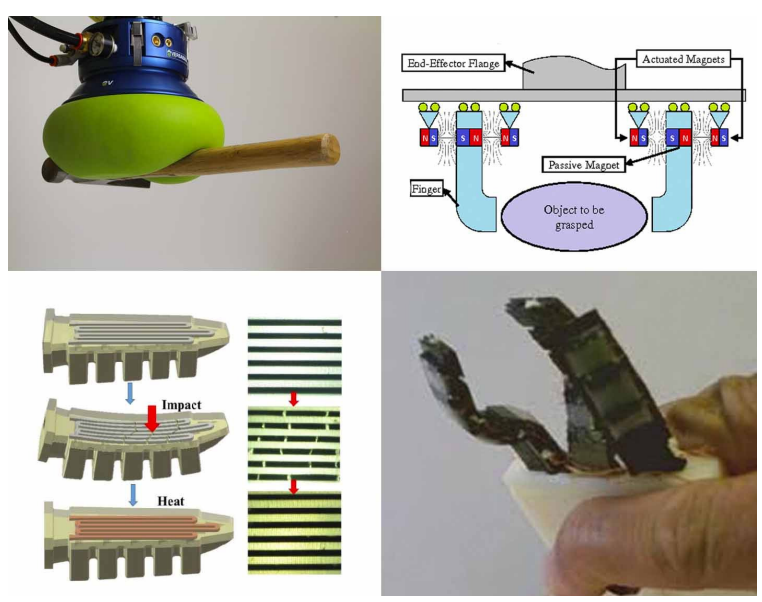

Figura 3: Pinzas de resistencia variable

\section{METODOLOGÍA}

Antes de que la pinza sea diseñada se han de definir sus especificaciones. Debe resolver los problemas explicados en la sección anterior; es decir, cambiar la rigidez al instante y evitar el uso de elementos externos para cambiar la rigidez. Con este objetivo, se ha diseñado un dedo siguiendo el principio del Universal Gripper. En su diseño original, el Universal Gripper carece de destreza debido a su método de agarre, y no puede agarrar objetos grandes o pesados que requieren más de un punto de contacto, como bolsas o cajas, por lo que se requiere una actualización de su diseño 
básico. En resumen, la pinza debe superar todos estos problemas.

Para el diseño, se opta por utilizar una pinza paralela de dos dedos, ya que es el tipo de pinza más simple que puede realizar el agarre de objetos. Los dedos están diseñados para parecerse a la pinza universal, la activación se realiza con un servomotor y el movimiento se transmite a los dedos gracias a un husillo roscado.

La pinza se ha diseñado utilizando el software de modelado 3D SolidWorks ${ }^{\circledR}$ y luego se fabricó con una impresora 3D. Para las almohadillas universales en los dedos, se utilizan café y globos, para evitar que el café gotee, se atan los filtros al extremo del tubo que une el dedo. Las partes de los dedos se pueden ver en la fig. 4. Está formado por un globo lleno de café como parte principal, una cubierta impresa en 3D para el globo que le dará cierta estabilidad al dedo y otra pieza impresa en $3 \mathrm{D}$ que se unirá al eje roscado y la base de la pinza. Proporcionando movilidad al dedo.

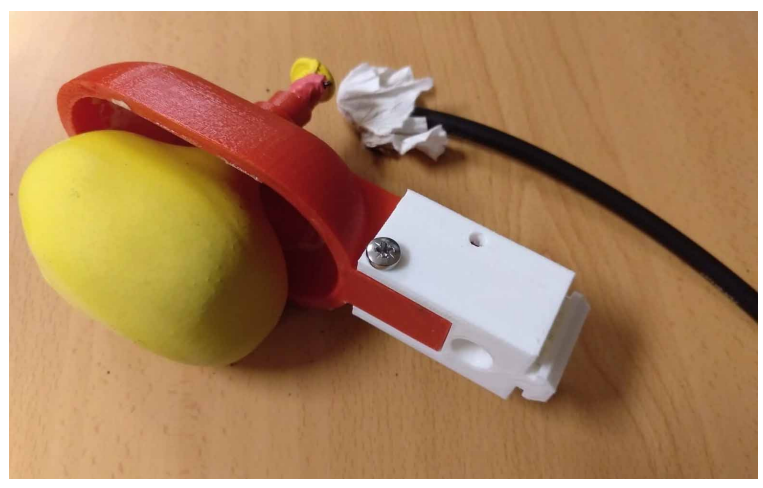

Figura 4: Partes del dedo

La base de la pinza se ha fabricado a partir de perfilería estructural de aluminio en el que todos los módulos están conectados, y también proporciona el riel para el movimiento del dedo. Aparte de los módulos de dedo, se ha diseñado un módulo de motor. Este sistema modular ayudará a cambiar rápidamente los dedos o los métodos de actuación, por lo que todas las mejoras y reemplazos de las piezas se pueden hacer más rápido y sin tener que cambiar la pinza desde cero.

Para tener otra pinza para comparar con la diseñada se fabricará un nuevo dedo, este dedo es similar al Festo ${ }^{\circledR}$ FinGripper [6], pero sin la parte de conexión interna. Todavía puede realizar algunas capacidades de deformación y adaptación en el agarre al fabricarse con un material rígido, pero con un dedo muy fino. Estos dedos también se pueden conectar a la pieza de unión, por lo que cambiar entre pinzas es una tarea rápida y fácil. En la fig. 5 se pueden ver ambas pinzas ya montadas.

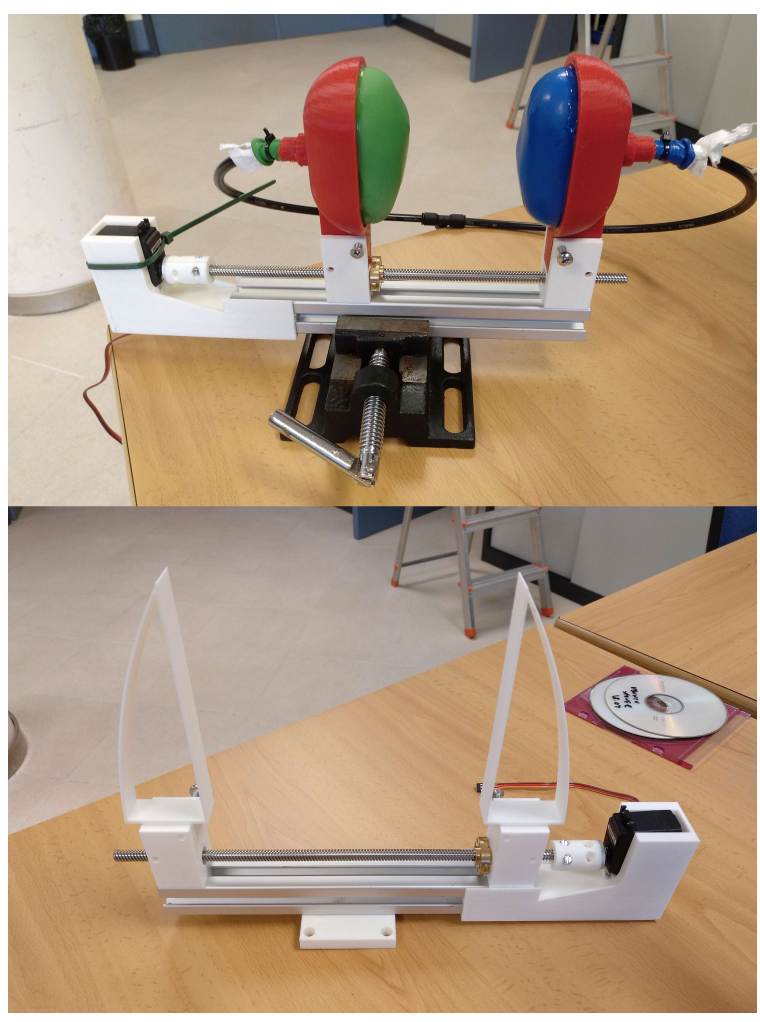

Figura 5: Ambas pinzas

\section{EXPERIMENTOS}

En primer lugar, la pinza se monta en un brazo robótico Motoman ${ }^{\circledR}$, como se puede ver en la fig. 6. Esto se hace para poder realizar una serie de agarres en 5 objetos diferentes. El objetivo del experimento es evaluar el rendimiento de agarre de la pinza. Para poder realizarlo, agarrará un objeto, luego lo elevará en el aire y el último paso será agregar una perturbación en el agarre moviendo manualmente el objeto mientras se mantiene entre los dedos. Este proceso se repetirá diez veces con cada objeto. Si el proceso completo se realiza sin que el objeto caiga, el agarre recibirá una puntuación de 3 , si el objeto cae en la perturbación una puntuación de 2 , si el objeto no pudo levantarse pero el agarre está bien realizado, se obtiene una puntuación de 1, y finalmente si el agarre no está bien realizado, la puntuación de 0 . La puntuación final de la pinza se calcula por la media de los diez intentos. Esto añade un nuevo objetivo para la pinza. La pinza diseñada debería poder obtener una puntuación mejor que 2.5 en todos los objetos que se agarran.

Los objetos seleccionados se tomaron del conjunto oficial para el Amazon Robotics Challenge 2017. El motivo es que están disponibles y son bien conocidos por la comunidad, y ya se han utilizado en diferentes bancos de prueba. 


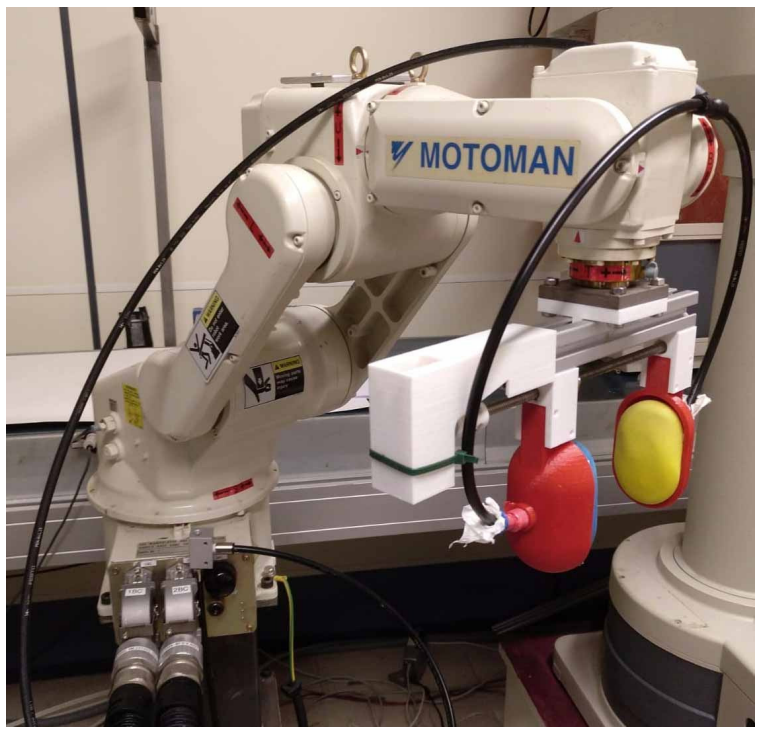

Figura 6: Pinza montada en un brazo robótico

Los cinco objetos seleccionados utilizados en los experimentos se pueden ver en la fig. 7, y el motivo de la selección para cada uno de los objetos es:

- Esponja (Esp): Este objeto se ha seleccionado debido a su forma prismática regular y sus altas capacidades de deformación. Si la pinza puede agarrar este objeto, demostrará su capacidad para tratar con objetos blandos simples.

- Pelotas de tenis (Ten): Este objeto se ha seleccionado porque es un objeto cilíndrico y apenas deformable. Por lo que es un objeto típico en el agarre clásico. La pinza debe al menos ser capaz de agarrar este objeto debido a su forma y propiedades regulares.

- Escobilla de baño (Esc): Este objeto se ha seleccionado por el mango y su forma alargada. Cuando se agarra este objeto, genera alta inestabilidad y un gran momento debido a su forma. Agarrar este tipo de objeto demostrará que la pinza puede soportar objetos similares a herramientas que generan inestabilidades al agarrar.

- Sales de Epsom (Sal): Este objeto se ha seleccionado debido a su alto peso, alta capacidad de deformación y por ser muy resbaladizo. Por lo que es un obejto altamente difícil de agarrar. Si la pinza logra agarrar las sales de Epsom, mostrará que tiene buenos resultados con objetos que son simultáneamente pesados, deformables y resbaladizos.

- Cucharas de medir (Cuc): Este objeto ha sido seleccionado debido a su forma, es un objeto articulado compuesto de múltiples partes rígidas que se unen con un anillo. Y también porque son bastante pequeños, por lo que la dificultad de agarre es alta.

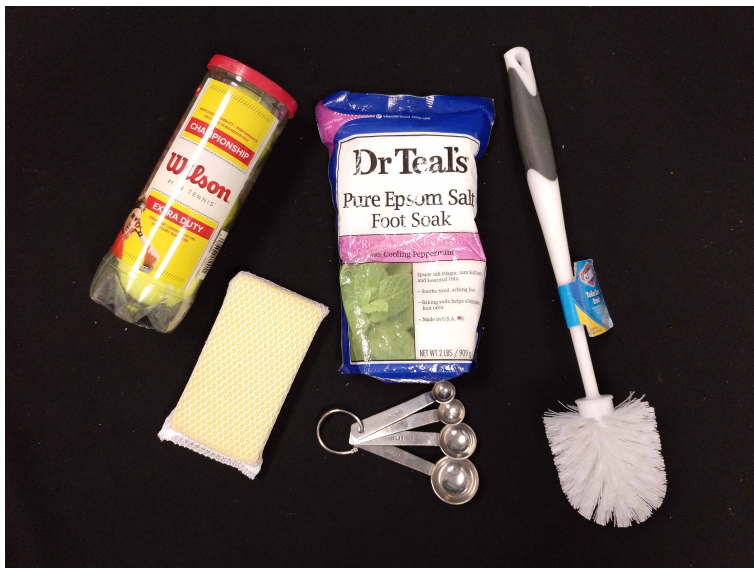

Figura 7: Objetos para los experimentos

Los experimentos fueron realizados por ambas pinzas. Para la pinza de rigidez variable, la prueba se realizará de dos maneras diferentes. La primera es utilizar la pinza sin activar la rigidez neumática variable. Al igual que una pinza paralela normal con algunas capacidades de adaptación proporcionadas por la dinámica pasiva del dedo. La segunda forma es utilizar la capacidad total de la pinza para cambiar su rigidez con el sistema neumático. Esto mostrará si es realmente mejor para el agarre cambiar la rigidez de la pinza que utilizarla como una pinza normal.

La fig. 8 muestra una representación visual de todos los pasos. Primero se coloca el objeto entre los dedos (arriba a la izquierda). Luego, los dedos comienzan a acercarse al objeto y realizan un agarre (arriba a la derecha). Después de que el agarre está terminado y asegurado, el objeto se levanta (abajo a la izquierda), luego se realiza una serie de perturbaciones manuales en el objeto y en la pinza para comprobar si el agarre puede soportarlas (abajo a la derecha). Finalmente el objeto se coloca en el suelo y se suelta.

\section{RESULTADOS}

Después de realizar los experimentos se anotan los resultados de los intentos de agarre en una tabla para cada pinza. Para analizar los resultados lo vamos a hacer pinza por pinza. Primero el FinGripper. Luego las pruebas con la pinza diseñada pero sin usar todo el sistema neumático. Finalmente la pinza diseñada con el sistema neumático.

En primer lugar, en la tabla 1 se pueden ver los resultados de los intentos de agarre de FinGripper. En la tabla se puede ver que la pinza tiene con- 


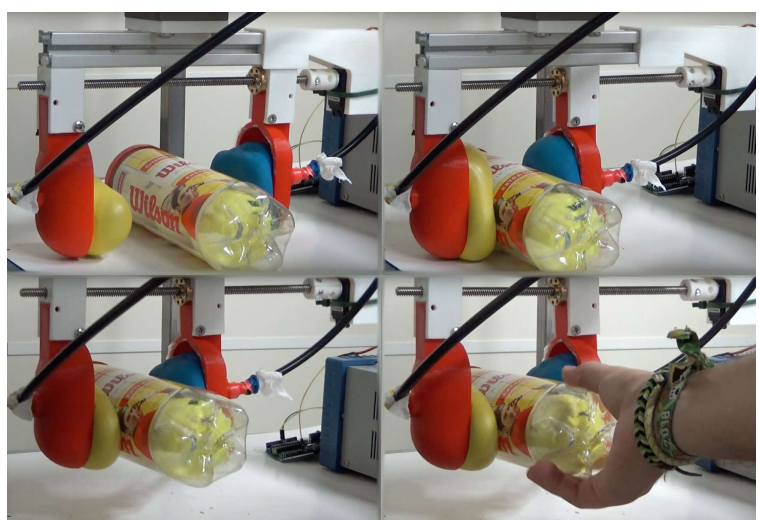

Figura 8: Grasping steps

Tabla 1: Resultados del FinGripper

\begin{tabular}{|c|c|c|c|c|}
\hline Esp & Ten & Esc & Sal & Cuc \\
\hline \hline 3 & 3 & 2 & 1 & 2 \\
\hline 3 & 3 & 2 & 1 & 1 \\
\hline 3 & 3 & 2 & 1 & 1 \\
\hline 3 & 3 & 2 & 1 & 0 \\
\hline 3 & 3 & 2 & 1 & 1 \\
\hline 3 & 3 & 2 & 1 & 1 \\
\hline 3 & 3 & 2 & 1 & 2 \\
\hline 3 & 3 & 2 & 1 & 2 \\
\hline 3 & 3 & 2 & 1 & 0 \\
\hline 3 & 3 & 2 & 1 & 1 \\
\hline
\end{tabular}

stantemente los mismos resultados para cada objeto individual, excepto las cucharas de medir. Así que es una pinza para la que generalmente es fácil predecir qué objeto puede o no puede agarrar. Si bien tiene buenos resultados para los objetos normales, cuando se trata de objetos más irregulares o pesados la pinza comienza a perder su eficiencia.

Tabla 2: Resultados de la pinza sin activación neumática

\begin{tabular}{|c|c|c|c|c|}
\hline Esp & Ten & Esc & Sal & Cuc \\
\hline \hline 2 & 3 & 3 & 3 & 0 \\
\hline 3 & 3 & 2 & 1 & 1 \\
\hline 3 & 3 & 3 & 1 & 3 \\
\hline 0 & 3 & 1 & 3 & 0 \\
\hline 3 & 2 & 2 & 3 & 3 \\
\hline 3 & 3 & 3 & 2 & 0 \\
\hline 2 & 3 & 3 & 3 & 0 \\
\hline 2 & 3 & 3 & 3 & 1 \\
\hline 3 & 3 & 2 & 1 & 1 \\
\hline 3 & 3 & 3 & 1 & 0 \\
\hline
\end{tabular}

En segundo lugar, en la tabla 2 se puden ver los resultados proporcionados por la pinza diseñada sin utilizar el sistema neumático para cambiar la rigidez de los dedos. En la tabla se puede ver que la pinza tiene buenos resultados de agarre en general, incluso sin cambiar la rigidez. Falla cuando se trata de agarrar las cucharas medidoras, y solo se logra un buen agarre cuando la posición de las cucharas es propicia.

Tabla 3: Resultados de la pinza con activación neumática

\begin{tabular}{|c|c|c|c|c|}
\hline Esp & Ten & Esc & Sal & Cuc \\
\hline \hline 3 & 3 & 3 & 3 & 3 \\
\hline 3 & 3 & 3 & 3 & 1 \\
\hline 3 & 3 & 3 & 3 & 3 \\
\hline 3 & 3 & 3 & 3 & 2 \\
\hline 3 & 3 & 3 & 3 & 2 \\
\hline 3 & 3 & 3 & 3 & 3 \\
\hline 3 & 3 & 3 & 3 & 3 \\
\hline 3 & 3 & 3 & 3 & 3 \\
\hline 3 & 3 & 3 & 3 & 3 \\
\hline 3 & 3 & 3 & 3 & 3 \\
\hline
\end{tabular}

Finalmente, en la tabla 3 se muestran los resultados de agarre de la pinza diseñada utilizando el sistema neumático para cambiar la rigidez. La pinza completa tiene mejores resultados que las anteriores, lo que resulta en un rendimiento perfecto para todos los objetos, excepto las cucharas de medición, ya que es el objeto más difícil de agarrar.

\section{DISCUSIÓN}

Para obtener una mejor comparación de los resultados en la tabla 4 se muestra la puntuación como una media de todos los intentos de agarre por agarrador y objeto. En la tabla aparecen los nombres de las pinzas como:

- FGrip: Pinza diseñada con base en el FinGipper de Festo ${ }^{\circledR}$.

- NGrip: Pinza diseñada sin utilizar el sistema neumático para cambiar su propia rigidez.

- PGrip: Pinza diseñada utilizando el sistema neumático para cambiar su propia rigidez.

Tabla 4: Resultados finales

\begin{tabular}{|c||c|c|c|}
\hline & FGrip & NGrip & PGrip \\
\hline Esponja & 3 & 2.4 & 3 \\
\hline Pelotas de tenis & 3 & 2.9 & 3 \\
\hline Escobilla & 2 & 2.5 & 3 \\
\hline Sales de Epsom & 1 & 2.1 & 3 \\
\hline Cucharas & 1.1 & 0.9 & 2.6 \\
\hline
\end{tabular}


De los resultados en la sección anterior se puede observar que la pinza diseñada muestra buenos resultados incluso sin activar el sistema neumático para cambiar la rigidez. Al menos para la esponja, y la escobilla, habiendo superado el objetivo de obtener más de 2,5 en estos objetos. Y con un 2,4 en las pelotas de tenis.

Cuando la pinza utiliza el sistema neumático la puntuación de agarre se convierte en perfecta para todos los objetos, excepto para las cucharas de medición. Esto puede deberse al diseño del dedo, ya que carece de una punta o uña al final para proporcionar agarres de precisión. En todos los objetos la pinza con el sistema neumático activado supera el objetivo de tener al menos 2,5 como puntación promedio.

En la fig. 9 se muestran ambas pinzas agarrando las pelotas de tenis para tener una comparación visual de cómo se realiza el agarre para ambas. Para la pinza principal diseñada la capacidad de agarre se basa en la deformación y adaptación de las almohadillas de café en los dedos. Mientras que el FinGripper utiliza todo el dedo para adaptarse al objeto mientras agarra, debido a las propiedades de deformación del diseño del dedo, tal como aparece en la figura.

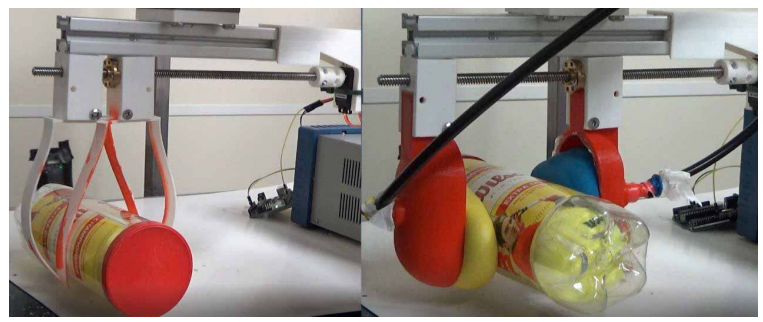

Figura 9: Ambas pinzas cogiendo las pelotas de tenis

Al comparar los resultados de ambas pinzas, se aprecia una diferencia significativa. Si bien el FinGripper obtiene buenos resultados con los objetos normales, carece de eficiencia cuando se trata de los objetos que son más difíciles de agarrar.

La pinza diseñada supera el objetivo establecido de obtener una puntuación media mayor a 2,5 para cada objeto, y también la superación de los objetivos establecidos para las pinzas de rigidez variable. Con el sistema neumático puede cambiar inmediatamente su propia rigidez a voluntad, y tiene un diseño fácil y sencillo que también permite un control fácil y sencillo.

\section{CONCLUSIÓN}

En este artículo se ha presentado un nuevo tipo de pinza paralela con accionamiento de rigidez vari- able. La pinza está formada por dos dedos paralelos con un movimiento horizontal provisto por un servomotor y una varilla roscada. El diseño de los dedos se basa en la pinza universal [10], por lo que se pueden adaptar a la forma del objeto y las posibles irregularidades. Logrado gracias a las capacidades únicas de la pinza universal.

En la fig. 10 se pueden ver los pasos para realizar un agarre con la pinza. Primero, el objeto se coloca entre los dedos (arriba a la izquierda), las cavidades de los dedos reciben una pequeña presión positiva para que los dedos se ablanden y puedan cubrir mejor el objeto. Luego los dedos realizan el agarre (arriba a la derecha). Una vez que se realiza el agarre, en lugar de presión positiva lo cambiamos a negativo (centro-izquierda), por lo que se asegura el agarre. Posteriormente, se levanta el objeto (centro-derecha) y se prueba el agarre mediante perturbaciones manuales (parte inferior izquierda). Finalmente el objeto se coloca en el suelo y se suelta. En la última imagen (abajo a la derecha) podemos ver que la forma del objeto sigue en el dedo hasta que se libera el vacío de la cavidad.

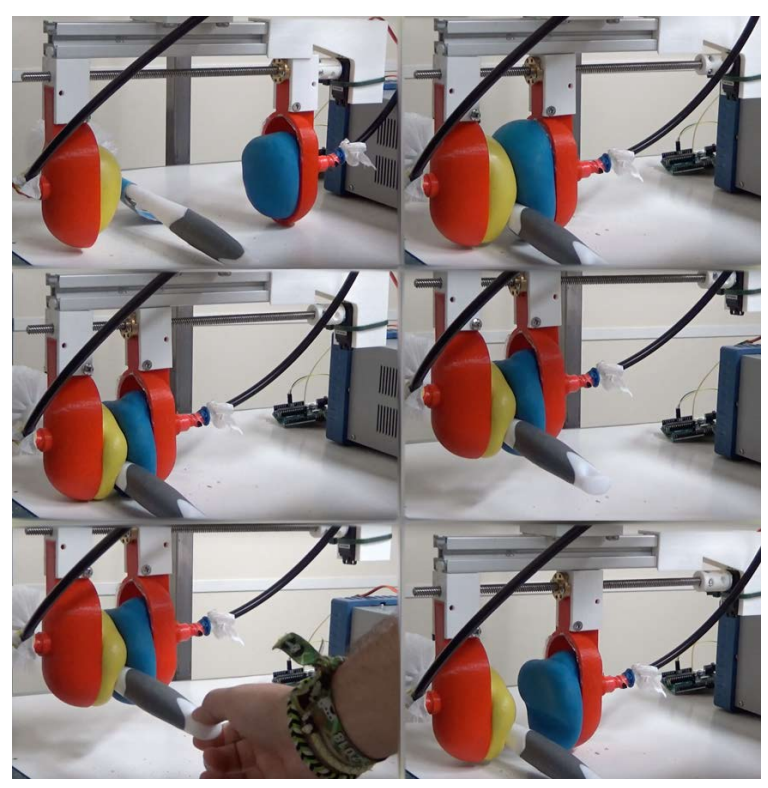

Figura 10: Proceso de agarre

La pinza de rigidez variable ha superado los problemas que habían surgido anteriormente para otro tipo de diseños. Estos problemas son la falta de inmediatez al cambiar diferentes parámetros de rigidez y la complejidad de los sistemas utilizados para cambiar las propiedades de las uniones. De hecho, la pinza puede cambiar su rigidez casi instantáneamente gracias al sistema neumático, y tiene un diseño simple. La estructura modular de la propia pinza le permite cambiar o mejorar sus elementos de una manera rápida y fácil, sin tomar 
demasiado tiempo en las reparaciones o tener que hacer una nueva pinza desde cero después de cada pequeño fallo.

En futuras investigaciones se seguirá mejorando el diseño de la pinza para obtener mejores resultados, haciéndolo más pequeño reduciendo el volumen de los dedos actuales o agregando elementos tales como uñas para mejorar su aptitud para agarres de precisión. Con estas mejoras se probarán las nuevas pinzas con objetos adicionales de Amazon Robotic Challenge 2017. El objetivo será alcanzar el máximo número de objetos con una sola pinza cambiando la rigidez o el modo y la estrategia de agarre. Se podrían usar versiones más elaboradas para implementar la rigidez variable de manos con varios dedos, como la mano de Barret [14] o la mano de Schunk.

\section{Agradecimientos}

Este artículo describe la investigación realizada en UJI RobInLab. El apoyo para esta investigación fue proporcionado por el Ministerio de Economía y Competitividad (DPI2015-69041-R).

\section{English summary}

\section{DESIGN OF A NOVEL VARIABLE-STIFFNESS GRIP- PER USING JAMMING FINGERS}

\author{
Abstract \\ This paper addresses the design of a \\ variable-stiffness gripper to solve a prob- \\ lem that arises in online retail stores, in \\ which huge warehouses keep all the stock. \\ In the process of automating the task of \\ picking individual objects, reliable grasp- \\ ing is a major issue due to the uncer- \\ tainty of the objects shape and position. To \\ address this problem, we have designed a \\ novel variable-stiffness gripper capable of \\ grasping different types of objects by using \\ and adapting the technology of the univer- \\ sal gripper in a 2-finger parallel gripper. \\ To test the gripper five different objects \\ have been lifted and manipulated with it \\ and compared with another simple gripper \\ design based on Festo ${ }^{\circledR}$ FinGripper. Our \\ experiments show that this gripper, with \\ variable-stiffness actuation, improves the \\ grasping capabilities of a gripper without \\ the variable-stiffness ability.
}

Keywords: Variable-stiffness, Robotic gripper, Jamming.

\section{Referencias}

[1] A. Firouzeh, J. Paik, "An under-actuated origami gripper with adjustable stiffness joints for multiple grasp modes", in Smart Mater. Struct., vol. 26, no. 5, 2017.

[2] A.H. Memar, N. Mastronarde, E.T. Esfahani, "Design of a Novel Variable Stiffness Gripper Using Permanent Magnets", in IEEE Int. Conf. Robot. Autom., 2017, pp. 2818-2823.

[3] A. Mo and W. Zhang, "Pin array hand: A universal robot gripper with pins of ellipse contour," 2017 IEEE International Conference on Robotics and Biomimetics (ROBIO), Macau, 2017, pp. 2075-2080.

[4] A. Rodić, B. Miloradović, S. Popić, S. Spasojević, B. Karan, "Development of Modular Compliant Anthropomorphic Robot Hand", in: Pisla D., Bleuler H., Rodic A., Vaida C., Pisla A. (eds) New Trends in Medical and Service Robots. Mechanisms and Machine Science, vol 16. Springer, Cham., 2014.

[5] A.P. del Pobil et al., "UJI RobInLab's approach to the Amazon Robotics Challenge 2017", presented at the IEEE International Conference on Multisensor Fusion and Integration for Intelligent Systems (MFI), Daegu, Korea, Nov 2017 16-18.

[6] C. Whitney, V. Gabrielle, O. Maeve, M. William and R. Chris, "Fin Ray® Effect Inspired Soft Robotic Gripper: From the RoboSoft Grand Challenge toward Optimization", in: Frontiers in Robotics and AI, vol. 3,2016

[7] D. Rus, T. Tolley, "Design, fabrication and control of soft robots", in: Nature, vol. 521, no. 7553,2015 , pp. $467-475$.

[8] H. Yufei, W. Tianmiao, F. Xi, Y. Kang, M. Ling, G. Juan, W. Li, "A Variable Stiffness Soft Robotic Gripper with Low-MeltingPoint Alloy", in Proceedings of the 36th Chinese Control Conference, 2017, pp. 6781-6786 .

[9] J. Hughes, U. Culha, F. Giardina, F. Guenther, A. Rosendo and F. Iida, "Soft Manipulators and Grippers: A Review", in: Frontiers in Robotics and AI, vol. 3, 2016, pp. 69. 
[10] J. R. Amend, E. Brown, N. Rodenberg, H. M. Jaeger and H. Lipson, "A Positive Pressure Universal Gripper Based on the Jamming of Granular Material," in IEEE Transactions on Robotics, vol. 28, no. 2, pp. 341-350, April 2012.

[11] M. Manti, V. Cacucciolo, M. Cianchetti, "Stiffening in soft robotics: A review of the state of the art", in IEEE Robot. Automat. Mag. 23, 2017, pp. 93-106.

[12] S. Licht, E. Collins, D. Ballat-Durand and M. Lopes-Mendes, "Universal jamming grippers for deep-sea manipulation," OCEANS 2016 MTS/IEEE Monterey, Monterey, CA, 2016, pp. 1-5.

[13] T. Nishida, Y. Okatani and K. Tadakuma, "Development of Universal Robot Gripper Using MR $\alpha$ Fluid", International Journal of Humanoid Robotics, Vol. 13, No. 04, 2016.

[14] W.T. Townsend, "The Barrett Hand grasperprogrammably flexible part handling and assembly", Industrial Robot: An International Journal", vol. 10(3), 2010, pp. 181-188

[15] Y. Jiang, J. R. Amend, H. Lipson and A. Saxena, "Learning hardware agnostic grasps for a universal jamming gripper," 2012 IEEE International Conference on Robotics and $\mathrm{Au}-$ tomation, Saint Paul, MN, 2012, pp. 23852391.

[16] Y. Li, Y. Chen, Y. Yang, Y. Wei Y, "Passive Particle Jamming and Its Stiffening of Soft Robotic Grippers", in: IEEE Trans. Robot., vol. 33 , no. 2 , 2017, pp. $446-455$.

[17] Y. Yang, Y. Chen, "3D printing of smart materials for robotics with variable stiffness and position feedback", presented in IEEE/ASME Int. Conf. Adv. Intell. Mechatronics (AIM), 2017, pp. 418-423.

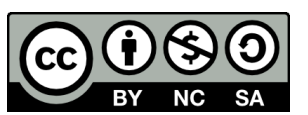

(C) 2019 by the authors. Submitted for possible open access publication under the terms and conditions of the Creative Commons Attribution CC BY-NC-SA 4.0 license (https://creativecommons.org/licenses/by-ncsa/4.0/deed.es). 